
d'urgence

Martine Lafrance, MSc

Nicole Leduc, PhD nuer la fréquentation des urgences des hôpitaux. Il devait constituer une nouvelle porte d'entrée vers les services de santé, en offrant à la population des conseils téléphoniques relatifs aux soins et aux ressources disponibles afin d'éviter le recours inapproprié aux services formels. Ce service, dispensé par des infirmières, est disponible 24 heures par jour, 7 jours par semaine, et permettrait de répondre à moindre coût aux besoins de santé de la population. ${ }^{1,2}$ Des services semblables de triage téléphonique nursing ont récemment vu le jour aux États-Unis, au Royaume-Uni et ailleurs au Canada, visant aussi à diminuer le recours aux services formels. ${ }^{3-6}$

Afin d'y arriver, il importe de leur assurer la plus grande notoriété possible. Comme ils visent entre autres à réduire le recours à l'urgence des hôpitaux pour des problèmes de santé non urgents, il serait intéressant de comparer la connaissance qu'en ont les usagers de l'urgence hospitalière avec celle des usagers d'autres types de services d'urgence. Comme ils constituent la principale clientèle visée, il apparaît important d'identifier au sein de cette clientèle les usagers auxquels devront plus spécifiquement s'adresser les futures campagnes de promotion d'Info-Santé CLSC.

Les objectifs de l'étude sont :

1)Identifier la proportion d'usagers de l'urgence hospitalière et des cliniques sans rendez-vous (privées et CLSC) qui connaissent le service téléphonique InfoSanté CLSC et l'ont déjà utilisé.

2) Identifier les principaux déterminants de la connaissance du service téléphonique.

\title{
Modèle théorique
}

Le modèle de la connaissance d'Info-Santé CLSC est illustré à la figure 1. Il a été développé à partir de trois modèles ou théories servant à expliquer le processus de recherche d'information, ${ }^{7}$ de prise de décision complexe ${ }^{8,9}$ et de diffusion d'une innovation. ${ }^{10}$

La connaissance d'Info-Santé CLSC

The translation of the Abstract appears at the end of the article.

Groupe de recherche interdisciplinaire en santé, Université de Montréal

Correspondance et demandes d'autorisation de reproduction : Martine Lafrance et Nicole Leduc, Groupe de recherche interdisciplinaire en santé, Faculté de médecine, Université de Montréal, C.P. 6128 succursale Centre-ville, Montréal (Québec) H3C 3J7, Tél. : 514-343-5633, Téléc. : 514-343-2207, Courriel : martine.lafrance@sympatico.ca

Organismes subventionnaires: Conseil de recherches médicales du Canada et Fonds de la recherche en santé du Québec

constitue la première étape du processus de diffusion d'une innovation. ${ }^{10}$ Elle serait influencée par cinq groupes de variables indépendantes :

- Caractéristiques sociodémographiques : l'âge du répondant, son genre, son 
revenu, sa scolarité, son emploi, la personne à qui s'adresse la consultation.

- Réseau social : quelqu'un à qui parler en cas de besoin, quelqu'un sur qui compter, le nombre de personnes habitant avec le répondant.

- Perception de la santé (excellente, très bonne, bonne, passable, moyenne, très mauvaise).

- Recherche d'information sur les services de santé : le besoin perçu d'information ("Croyez-vous être bien informé sur les services de santé sans rendez-vous près de chez vous? " oui ou non), la connaissance des services de santé, la disponibilité perçue de l'information. La connaissance et la disponibilité perçue ont été mesurées au moyen de trois questions chacune.

- Utilisation des services de santé : le service utilisé lors de l'étude (urgence d'hôpital, clinique privée sans rendezvous, CLSC sans rendez-vous), l'utilisation antérieure de ce service (oui ou non), la fréquentation des services médicaux durant les six derniers mois (nombre total), la disponibilité d'une source régulière de soins (non, oui sur rendezvous seulement, oui même sans rendezvous).

\section{METHODES}

La population à l'étude est constituée des adultes qui se présentent 1) soit à l'urgence d'un hôpital de soins de courte durée et dont l'état est considéré non urgent par l'infirmière au triage, 2) soit à l'une des trois cliniques médicales sans rendez-vous situées près de cet hôpital ou à un CLSC avoisinant offrant des services médicaux sans rendez-vous.

L'étude s'est déroulée de novembre 1997 à juin 1998 auprès de 402 patients de l'urgence hospitalière, 112 patients de chaque clinique médicale et 112 patients du CLSC $(n=850)$. Les patients ont été recrutés au hasard, dans la salle d'attente. Les informations ont été recueillies au moyen d'une entrevue structurée d'environ 15 minutes. Les taux de refus ont été respectivement de 55,6 \% pour l'hôpital et de $36,6 \%$ pour les autres services. Lors de l'étude, les deux salles d'attente de l'urgence hospitalière étaient fermées pour faire place aux patients sur civière, limitant de beaucoup le nombre de places assises.

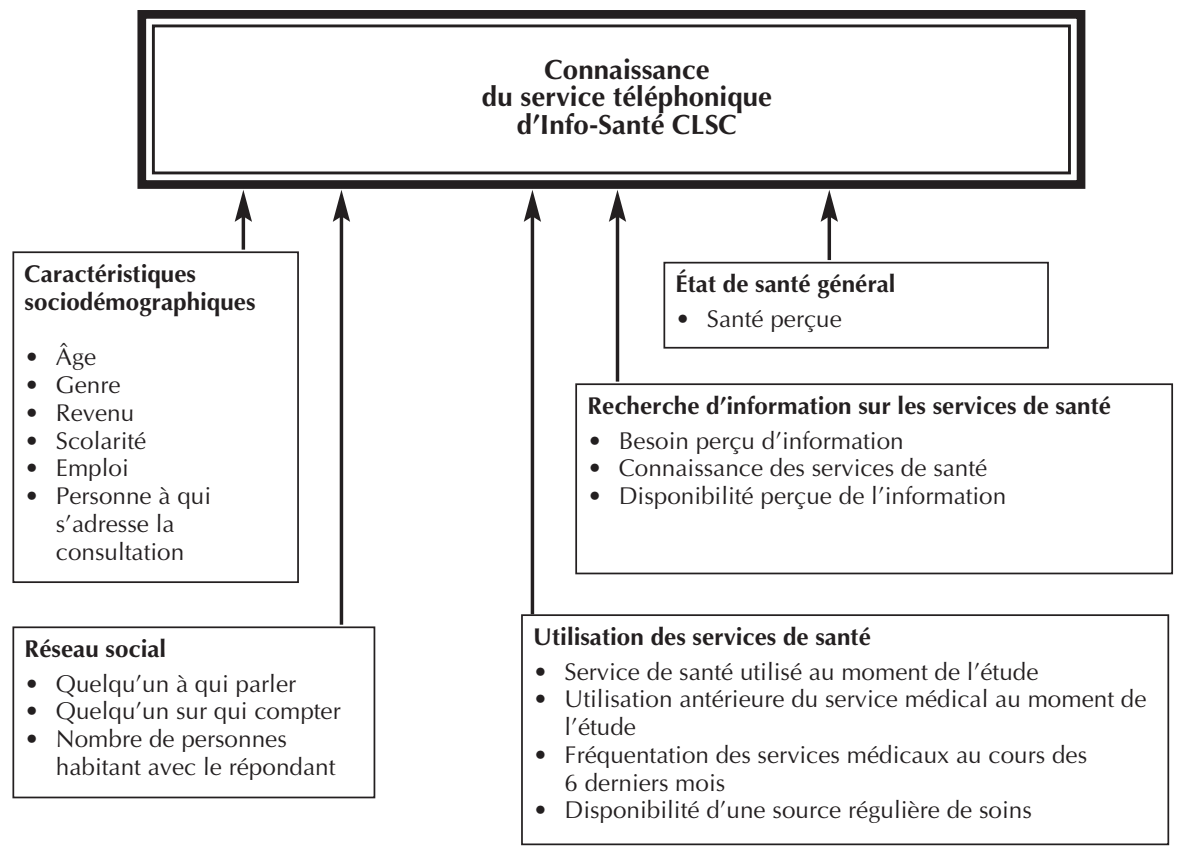

Figure 1. Modèle de la connaissance du service téléphonique Info-Santé CLSC

Une très forte proportion de patients ont alors refusé de se déplacer pour participer à l'entrevue afin de ne pas perdre leur siège. Les autres motifs de refus sont le manque d'intérêt, la durée de l'entrevue ou l'inconfort dû au problème de santé; ces motifs se retrouvaient dans des proportions équivalentes chez les patients de l'urgence hospitalière et ceux des cliniques.

Les analyses ont consisté en des statistiques descriptives, de l'estimation par intervalle et des régressions logistiques multiples. Ces dernières ont été effectuées auprès des 768 patients pour lesquels nous disposions de données complètes.

\section{RÉSULTATS}

Le tableau I présente les principales caractéristiques des participants.

\section{La connaissance}

La majorité des patients (75,5\%; IC. $\left._{95}=0,73-0,78\right)$ connaissent InfoSanté CLSC. Pour les patients de l'urgence de l'hôpital, la connaissance est de 70,6 \% (IC. $\left._{95}=0,66-0,75\right)$, alors qu'elle est de $77,2 \%$ (IC. $\left._{95}=0,73-0,82\right)$ chez les patients des cliniques médicales et $87,3 \%$ (IC. ${ }_{95}=0,81-0,93$ ) chez ceux du CLSC.

La plupart des personnes connaissant le service l'ont déjà utilisé $(74,4 \%$; IC. $\left._{95}=0,71-0,78\right)$. Une proportion de $75,8 \%\left(\right.$ IC. $\left._{95}=0,71-0,81\right)$ proviennent de l'urgence hospitalière, $72,3 \%$ (IC. $_{95}=$
0,67 - 0,78) des cliniques médicales sans rendez-vous et 76,0\% $\left(\right.$ IC. $_{95}=0,67-$ 0,85) du CLSC.

\section{Les déterminants de la connaissance}

La connaissance serait influencée par l'âge, le genre, le revenu, l'utilisation des services sans rendez-vous du CLSC, la fréquentation des services médicaux depuis les six derniers mois, la disponibilité d'une source régulière de soins et la perception de l'état de santé (tableau II).

\section{DISCUSSION}

Notre étude a montré que 75,5\% des patients des services d'urgence connaissent Info-Santé CLSC et que, parmi ceux-ci, $74,4 \%$ y ont déjà eu recours. Très peu d'études semblent avoir examiné la notoriété de ce service. Un sondage réalisé en $1996^{11}$ auprès de 1402 adultes révèle qu'il était connu de $50 \%$ des personnes interrogées ; l'examen des données de ce sondage permet d'estimer à $17 \%$ la proportion d'adultes ayant utilisé ce service parmi ceux qui le connaissaient. Notre étude s'est déroulée quelque 12 à 18 mois plus tard, ce qui peut expliquer, en partie, la différence entre les proportions de personnes connaissant ce service. Comme notre étude portait sur des usagers de services médicaux, on pourrait penser que ces derniers sont davantage susceptibles de connaître un tel service. Les mêmes explications prévaudraient quant 
TABLEAU I

\section{Caractéristiques des répondants $(n=850)$}

\begin{tabular}{|c|c|c|}
\hline Caractéristiques & $\mathbf{n}$ & $\%$ \\
\hline \multicolumn{3}{|l|}{ Catégorie d'âge (ans) } \\
\hline$<20$ & 104 & 12, \\
\hline 20 à 29 & 191 & 23,0 \\
\hline 30 à 39 & 264 & 31 \\
\hline 40 à 49 & 131 & 15 \\
\hline 50 à 59 & 93 & 11 \\
\hline$\geq 60$ & 49 & 5 \\
\hline Moyenne & ans $\pm 13,5$ & \\
\hline \multicolumn{3}{|l|}{ Genre } \\
\hline Homme & 321 & 37 \\
\hline Femme & 529 & 62 \\
\hline \multicolumn{3}{|l|}{ Revenu total avant impôt } \\
\hline$<20000 \$$ & 180 & 22 \\
\hline Entre $20000 \$$ et $34999 \$$ & 197 & 24 \\
\hline Entre $35000 \$$ et $49999 \$$ & 157 & 19 \\
\hline $50000 \$$ ou plus & 268 & 33 \\
\hline \multicolumn{3}{|l|}{ Niveau de scolarité } \\
\hline Aucun ou primaire & 130 & 15 \\
\hline Secondaire & 353 & 42 \\
\hline Collégial & 229 & 27 \\
\hline Universitaire & 123 & 14 \\
\hline \multicolumn{3}{|l|}{ Emploi } \\
\hline Occupation à temps plein & 439 & 52 \\
\hline Occupation à temps partiel & 189 & 22 \\
\hline Aucune occupation (congé, retraite, sans emploi) & 206 & 24 \\
\hline \multicolumn{3}{|l|}{ Personne à qui s'adresse la consultation } \\
\hline Répondant lui-même & 645 & 77 \\
\hline Son enfant & 179 & \\
\hline Autre (parent, conjoint, neveu) & 7 & \\
\hline
\end{tabular}

TABLEAU II

Déterminants de la connaissance du service téléphonique Info-Santé CLSC chez les usagers des urgences hospitalières et des cliniques médicales sans rendez-vous $(n=768)$

\begin{tabular}{|c|c|c|c|}
\hline Caractéristiques & $\mathbf{R C}_{\text {ajusté }}$ & IC à $95 \%$ & $\mathbf{p}$ \\
\hline \multicolumn{4}{|l|}{ Caractéristiques sociodémographiques } \\
\hline Âge & 0,9678 & $0,9544-0,9815$ & 0,0000 \\
\hline \multicolumn{4}{|l|}{ Genre } \\
\hline Homme & 1 (référence) & & \\
\hline Femme & 3,4488 & $2,3519-5,0573$ & 0,0000 \\
\hline \multicolumn{4}{|l|}{ Revenu total avant impôt } \\
\hline$<20000 \$$ & 1 (référence) & & \\
\hline Entre $20000 \$$ et $34999 \$$ & 1,7205 & $1,0318-2,8689$ & 0,0375 \\
\hline Entre $35000 \$$ et $49999 \$$ & 3,0622 & $1,6931-5,5382$ & 0,0002 \\
\hline $50000 \$$ ou plus & 2,6275 & $1,5577-4,4320$ & 0,0003 \\
\hline \multicolumn{4}{|l|}{ Utilisation des services de santé } \\
\hline \multicolumn{4}{|l|}{ Service de santé utilisé au moment de l'étude } \\
\hline Urgence de I'hôpital & 1 (référence) & & \\
\hline Clinique médicale sans rendez-vous & 1,4193 & $0,9554-2,1085$ & 0,0829 \\
\hline CLSC sans rendez-vous & 4,0485 & $1,9585-8,3686$ & 0,0002 \\
\hline Fréquentation des services médicaux & & & \\
\hline au cours des 6 derniers mois & 1,0892 & $1,0236-1,1590$ & 0,0070 \\
\hline \multicolumn{4}{|l|}{ Disponibilité d'une source régulière de soins } \\
\hline Pas de source régulière de soins & 1 (référence) & & \\
\hline Source régulière disponible avec r.v.* & 2,0054 & $1,1674-3,4449$ & 0,0117 \\
\hline Source régulière disponible sans r.v.* & 1,2776 & $0,8228-1,9838$ & 0,2752 \\
\hline \multicolumn{4}{|l|}{ État de santé général } \\
\hline \multicolumn{4}{|l|}{ Santé perçue } \\
\hline Excellente & 1 (référence) & & \\
\hline Très bonne & 0,8798 & $0,5538-1,3979$ & 0,5878 \\
\hline Bonne & 0,4280 & $0,2573-0,7122$ & 0,0011 \\
\hline Passable & 0,4507 & $0,2188-0,9282$ & 0,0306 \\
\hline Moyenne ou très mauvaise & 1,3116 & $0,3637-4,7309$ & 0,6785 \\
\hline Paramètres statistiques & $\chi^{2}$ & dl & \\
\hline Test d'ajustement de Hosmer et Lemeshow & $\tilde{9}, 5088$ & 8 & 0,3012 \\
\hline
\end{tabular}

Valeur prédictive $=78,13 \%$ Sensibilité $=94,70 \%$

Spécificité $=26,00 \%$ pas reliés aux caractéristiques des patients, mais plutôt au contexte organisationnel de l'urgence prévalant au moment de l'étude. Un questionnaire auto-administré, plutôt qu'une entrevue, aurait peut-être favorisé davantage la participation.

Nos résultats démontrent que l'âge est un facteur associé négativement à la connaissance du service. Ils sont semblables à ceux d'autres études. ${ }^{2,11-13}$ L'âge est considéré par Lenz ${ }^{7}$ comme influençant défavorablement le comportement de recherche d'information. L'auteur émet l'hypothèse que la personne âgée a un grand bagage de connaissance et qu'elle n'aurait plus d'intérêt à mettre de l'énergie dans une nouvelle recherche d'information. De plus, ces personnes peuvent avoir moins de contacts avec la famille ou les amis que les personnes plus jeunes et leurs capacités cognitives diminuent au fil des années. ${ }^{14,15} \mathrm{La}$ personne doit trouver un avantage relatif à adopter une innovation tel Info-Santé CLSC : ce service peut-il apporter de meilleurs renseignements que ceux qu'elle possède déjà ? Sa complexité de fonctionnement peut de plus freiner la démarche de recherche d'information de la personne âgée. ${ }^{10,16}$

La probabilité de connaître Info-Santé CLSC serait plus élevée chez les femmes. Lors de la visite des femmes aux services d'urgence, la consultation s'adressait souvent à l'enfant plutôt qu'à la répondante elle-même. Plusieurs études indiquent que les parents d'enfants malades utilisent souvent les urgences hospitalières pour des soins non urgents. ${ }^{17-22}$ Le profil des usagers ayant déjà fait appel à Info-Santé CLSC dans les études antérieures, consiste majoritairement en des femmes demandant des informations pour leurs enfants ou leurs proches. ${ }^{2,9,13,23}$ Les parents sont fréquemment confrontés aux maladies de leurs enfants et ont parfois de la difficulté à trouver de l'information les aidant à mieux comprendre. ${ }^{24}$ Info-Santé CLSC semble être une source d'information à laquelle ils ont recours.

Le revenu influencerait positivement la probabilité de connaître Info-Santé. D'après SOM, ${ }^{11}$ ce service téléphonique est utilisé principalement par les femmes à la maison ou en chômage. Hagan, Morin et Lépine $^{2}$ ont trouvé que le revenu familial avant impôt était de $40000 \$$ ou moins pour les deux tiers des utilisateurs. Le taux à la différence observée entre la proportion d'utilisateurs d'Info-Santé CLSC dans la population générale et les usagers des services d'urgence.
Le taux de refus chez les patients de l'urgence hospitalière s'est avéré élevé, comparativement à ceux des cliniques. Cependant, les motifs de refus n'étaient 
de notoriété serait en outre plus bas chez les individus à faible revenu. ${ }^{12} \mathrm{La}$ différence entre nos résultats et ceux mentionnés pourrait être attribuable aux régions où les études ont été effectuées, ${ }^{13,23}$ réflétant des profils distincts de besoins sociosanitaires. D'après Lenz, ${ }^{7}$ les personnes ayant un statut socio-économique élevé recherchent davantage d'information. Elles présentent une bonne capacité cognitive leur facilitant la définition du besoin d'information et une plus grande confiance vis-à-vis les professionnels de la santé. Elles sont également intéressées aux sujets touchant la santé et ont accès à un large réseau social.

Notre étude montre qu'il existe une association entre les utilisateurs du service sans rendez-vous du CLSC et la connaissance d'Info-Santé CLSC comparativement à ceux de l'urgence hospitalière. Ceci s'explique entre autres par une plus grande exposition chez les usagers des CLSC à la publicité concernant le service téléphonique offert par l'organisation qu'ils fréquentent. Aucune différence significative n'a été observée entre les usagers des cliniques médicales et ceux de l'urgence hospitalière. De plus, l'utilisation antérieure d'Info-Santé CLSC était la même chez les usagers des trois types de service d'urgence. On peut se demander qu'elle est l'influence du service téléphonique sur le recours à l'urgence hospitalière pour des problèmes de santé non urgents, étant donné que la majorité des participants connaissent et utilisent le service. Il serait intéressant de vérifier dans quelle mesure ces patients ont contacté Info-Santé CLSC avant de se rendre à l'urgence, à quelle proportion Info-Santé CLSC a effectivement recommandé de consulter et combien ont suivi sa recommandation à l'égard du service d'urgence utilisé au moment de l'étude.

Par ailleurs, le nombre de visites à des services médicaux depuis les six derniers mois s'est avéré un déterminant de la connaissance du service téléphonique. Un volume élevé de visites à l'urgence hospitalière, aux cliniques médicales sans rendezvous, au CLSC ou au médecin, pourrait favoriser les échanges avec les intervenants en santé ou avec d'autres patients, augmentant ainsi les chances d'apprendre l'existence d'Info-Santé CLSC.

Les patients ayant une source régulière de soins disponible avec rendez-vous seule- ment, auraient une probabilité plus forte de connaître Info-Santé CLSC, résultant peut-être d'une recherche accrue d'information.

Une santé qualifiée en général de bonne ou passable plutôt qu'excellente diminuerait la probabilité de connaître InfoSanté CLSC. On peut penser que les gens décrivant leur santé comme bonne ou passable ont une plus forte probabilité de recourir fréquemment aux services de santé, développant ainsi certaines habitudes de consommation. Ils seraient par conséquent moins ouverts à connaître de nouvelles alternatives telles qu'Info-Santé CLSC.

\section{CONCLUSION}

Cette étude a permis d'identifier certains facteurs influençant la connaissance du service téléphonique Info-Santé CLSC. Elle a en outre montré que la majorité des patients des services d'urgence connaissent ce service et l'ont déjà utilisé. Elle s'est intéressée aux déterminants de la connaissance d'Info-Santé CLSC chez les usagers des services d'urgence. La connaissance d'une innovation telle qu'un service de triage téléphonique nursing constitue une première étape dans son adoption. Ses déterminants different fort probablement de ceux amenant les individus à l'utiliser de façon régulière. Il est donc nécessaire de les étudier de façon distincte.

Les résultats de notre étude permettront le développement de stratégies visant à accroître la notoriété d'Info-Santé CLSC par des campagnes de promotion ciblant prioritairement les personnes âgées, celles à faibles revenus, les hommes et les usagers des services d'urgence n'ayant pas de source régulière de soins. Il importe de poursuivre en parallèle l'évaluation de l'impact des services de triage téléphonique nursing, tel Info-Santé CLSC, sur le recours aux services de santé.

\section{RÉFÉRENCES}

1. Ministère de la Santé et des Services Sociaux. Accessibilité continue 24/7. Service téléphonique. Cadre de référence. Gouvernement du Québec. Direction générale des programmes. Québec, 1994.

2. Hagan L, Morin D, Lépine R. Évaluation provinciale des services Info-Santé CLSC : Perception des utilisateurs. Faculté des sciences infirmières. Centre de recherche sur les services communautaires. Université Laval. Québec, 1998.

3. Gallagher M, Huddart T, Henderson B. Telephone triage of acute illness by a practice nurse in general practice: Outcomes of care. $\mathrm{Br} \mathrm{J}$ Gen Pract 1998;Apr;48(429):1141-45.

4. O'Connell JM, Johnson DA, Stallmeyer J, Cokingtin D. A satisfaction and return-oninvestment study of a nurse triage service. $A m ~ J$ Manag Care 2001;Feb;7(2):159-69.

5. Williams S, Crouch R, Dale J. Providing healthcare advice by telephone. Prof Nur 1995;10(12):750-52.

6. Ministry of Health and Ministry Responsible for Seniors. Partnerships for Better Health: A selfcare pilot project. Final evaluation report. British Columbia: Capital Health Region, 2000.

7. Lenz ER. Information seeking: A component of client decision and health behavior. Advances in Nursing Science 1984;6(4):59-72.

8. Assael H. Consumer Behavior and Marketing Action, 2e édition. Boston: Kent Publishing Company, 1984

9. Assael H. Marketing. Forth Worth, Texas: Dryden Press, 1998.

10. Rogers EM. Diffusion of Innovations, Fourth Edition. New York: The Free Press, 1995.

11. SOM. Inc. Évaluation de la notoriété du service Info-Santé des CLSC de la Montérégie. Rapport présenté au CLSC du Richelieu (version préliminaire), Montréal, 1996.

12. Ministère de la Santé et des Services Sociaux. Évaluation provinciale des services Info-Santé CLSC. Rapport final 1994-1999. Direction de l'évaluation, de la recherche et de l'innovation. Direction des communications. Québec, 1999.

13. Hagan L, Garon G. Info-Santé. Région de Québec. Un service infirmier efficace? Évaluation de la satisfaction et de la capacité d'autosoin chez les usagers du service téléphonique Info-Santé de la région de Québec. Centre de recherche sur les services communautaires. Université Laval. Québec, 1996.

14. Bélanger S, Bouchard L. La recherche d'information : un risque calculé pour les personnes atteintes d'un cancer. L'infirmière du Québec $1998 ;(3): 30-40$

(continue à la page suivante)

\section{ABSTRACT}

Background: The CLSC's Info-Santé telephone service is one of the Quebec health network's reorganization measures aimed at decreasing the number of visits to hospital emergency rooms for nonurgent health problems. This study analyzes the awareness of this service among users of hospital emergency services and walk-in medical clinics.

Methods: In all, 850 patients were interviewed. Logistic regressions were performed.

Results: The results revealed that $75.5 \%(\mathrm{Cl} .95=0.73-0.78)$ of the participants were indeed aware of the CLSC's Info-Santé telephone service. Awareness was found to be dependent on age, sex, income, the use of walk-in CLSC medical services, the availability of a regular source of health care and the perception of one's health status.

Interpretation: Further strategies should be developed in order to increase the use of this service, thus helping to guide patients to more appropriate sources of health care. 
15. Chevarie A. Recension théorique sur les différents facteurs influençant la recherche d'aide. Centre de recherche sur les services communautaires. Université Laval. Québec, 1991.

16. Bandura A. Social Foundations of Thought and Action. A Social Cognitive Theory. New Jersey: Prentice-Hall, Inc., 1986.

17. Berns SD, et al. Appropriate use of a pediatric emergency department: Is the pediatrician called before the visit ? Pediatric Emergency Care 1994;10(2):13-17.

18. Chande VT, Krug SE, Warm EF. Pediatric emergency department utilisation habits: A consumer survey . Pediatric Emergency Care 1996;12(1):27-30

19. Chande VT, Wyss N, Exum V. Educational interventions to alter pediatric emergency department utilization patterns. Arch Pediatr Adolesc Med 1996;150(5):525-28

20. Quill TE, Brody H. Physician recommendations and patient autonomy: Finding a balance between physician power and patient choice. Ann Intern Med 1996;125(9):763-69.

21. Szilagyi PG. Managed care for children: Effect on access to care and utilization of health services. Future Child 1998;8(2):39-59.

22. Vivier PM, et al. Is continuity of care preserved in children who utilize the pediatric emergency department? Pediatrics 1995;95(1):37-41.

23. Pelletier D, Ouellet H. Développement du service d'accessibilité téléphonique continue-CLSC 24/7 des régions de Lanaudière et des Laurentides. Études prospectives du S.A.T. Rapport 1. Centre de recherche sur les services communautaires. Université Laval. Québec, 1994.

24. Kai J. Parents' difficulties and information needs in coping with acute illness in preschool children: A qualitative study. BMJ 1996;313(7063):987-90.

Reçu : 31 janvier 2001

Accepté : 22 juin 2001

\section{Coming Events Activités à venir}

To be assured of publication in the next issue, announcements should be received by February 1, 2002 and valid as of February 28, 2002. Announcements received after February 1, 2002 will be inserted as time and space permit.

Pour être publiés dans le prochain numéro, les avis doivent parvenir à la rédaction avant le $\mathbf{1}^{\text {er }}$ février 2002 et être valables à compter du 28 février 2002. Les avis reçus après le $1^{\text {er }}$ février 2002 seront insérés si le temps et l'espace le permettent.

$8^{\text {th }}$ Annual Qualitative Health Research Conference Sponsored by International Institute for Qualitative Methodology (IIQM) and University of Alberta 4-6 April 2002 Banff, AB Keynote speakers: Jeanie Kayser-Jones, Margaret Lock Contact:

IIQM

Tel: 780-492-9041

Fax: 780-492-9040

E-mail: qualitative.institute@ualberta.ca

www.ualberta.ca/ -iiqm/qhr2002/

6th National Health Promotion Conference Partnership Research for Health and Social Change The Community Health Promotion Coalition at the University of Victoria, in collaboration with the Canadian Consortium for Health Promotion Research

7-10 April 2002

www.hp2002.uvic.ca

Victoria, BC

$10^{\text {th }}$ Annual Canadian Association of Nurses in AIDS Care (CANAC) Conference

Social Justice: The Essence of HIVIAIDS Nursing

Featuring Stephen Lewis, UN Special Envoy on AIDS in Africa

14-17 April $2002 \quad$ Vancouver, BC

Contact:

CANAC/ACIIS 2002

Conference Secretariat

Tel: 604-983-3173

E-mail: candice@devonknight.com

Public/Private Strategies for the Funding \& Delivery of Health Care

The Canadian Institute

April 30 and May 1, 2002

Toronto, ON

Learn what some of the trailblazers in healthcare

administration and finance are doing to move health-

care delivery forward.

Contact:

Tel: 1-877-927-7936 (or 416-927-7936 in Toronto) Online at www.CanadianInstitute.com.

Evidence for Better Decision Making

Canadian Evaluation Society National Conference 2002

5-8 May 2002

Halifax, NS

Contact:

Catherine Lane

Tel: 902-424-7503Ｅ-mail: lanecl@gov.ns.ca www.evaluationcanada.ca

Health Policy, Practice and Research in the $21^{\text {st }}$ Century - Making a Difference

Organized by the Faculty of Health Sciences, Auckland University of Technology

6-9 May $2002 \quad$ Auckland City, New Zealand

The conference will have 2 themes: 1 ) the application of health research to health policy, health education/promotion and health practices; 2) highlighting some of the range of research methodologies used to address health policy, health education and health practice problems.

Contact:

Jim Borrows, Conference Coordinator

E-mail: jim.borrows@aut.ac.nz

www.aut.ac.nz/conferences/healthpolicy
ALCOA Forum 2002: A Lifelong Odyssey - Active Living for Older Adults

Saskatchewan Seniors Mechanism

9-12 May 2001

Regina, SK

Contact:

ALCOA office 1-800-549-9799 and/or the website: http://www.alcoa.ca or the Saskatchewan Seniors

Mechanism

Tel: 306-359-9956

$6^{\mathrm{e}}$ Conférence mondiale sur la Prévention et contrôle des traumatismes / 6th World Conference on Injury

Prevention and Control

Traumatismes, suicide et violence : construire un savoir, des politiques et des pratiques pour promouvoir un monde en sécurité / Injuries, Suicide and Violence:

Building Knowledge, Policies and Practices to Promote a Safer World

12-15 mai/May 2002

Montréal, QC

Contacter / Contact:

Congress Secretariat

511, place d'Armes, \# 600

Montréal, QC H2Y 2W7

Tel: $514-848-1133$ or 1-877-213-8368 (Canada \& US)

Fax: 514-288-6469

E-mail: trauma@coplanor.qc.ca

www.trauma2002.com

Canadian Dermatology Association

National Sun Awareness Week

13-20 May 2002

Contact:

613-730-6262 or $1-800-267-3376$

www.dermatology.ca

IIIrd European Conference on Travel Medicine

Travel \& Epidemics

Sponsored by WHO and CDC Atlanta

15-18 May 2002

Florence, Italy

Contact:

Prof. Walter Pasin

Director, WHO CC for Travel Medicine

Tel. +39-0541-2430

Fax. +39-0541-25748

E-mail: wpasini@rimini.com

$93^{\text {rd }}$ Annual Conference of the Canadian Public Health Association/93e conférence annuelle de l'Association canadienne de

santé publique

Our Environment, Our Health/ Notre environnement

notre santé

Co-sponsored by the Northwest Territories/Nunavut

Branch, CPHA/ Co-parrainée par la Division des

Territoires du Nord-Ouest et du Nunavut, ACSP

7-10 July/ juillet 2002 Yellowknife, NWT/ TN-O

Contact/ Contacter :

CPHA Conference Department

Département des conférences de l'ACSP

Tel/ Tél : 613-725-3769, ext. 126

Fax/ Téléc : 613-725-9826

E-mail/ Courriel : conferences@cpha.ca

www.cpha.ca 